\title{
Ensino clínico em cenários reais de prática
}

\section{Clinical teaching in the real practice settings}

\author{
Marcos C Borges ${ }^{1}$, Gustavo Frezza르, Cacilda da Silva Souza ${ }^{3}$, Valdes Roberto Bollela ${ }^{4}$
}

\begin{abstract}
RESUMO
O ensino clínico em cenários reais de prática é um componente essencial da educação na área da saúde. Ele estimula e garante que os estudantes aprendam sobre a prática profissional, sendo um dos mais efetivos meios para o desenvolvimento de habilidades clínicas, particularmente anamnese, exame físico, comunicação, ética, atitude humanística e profissionalismo. Tradicionalmente, enfermarias e ambulatórios, supervisionados por professores clínicos (preceptores), são os locais mais utilizados para o ensino clínico. Porém, apesar da excelente capacitação para a prática profissional, a grande maioria dos preceptores pode ter limitações para o ensino clínico. Assim, para que se atinja um nível de qualidade satisfatória no ensino clínico é necessário tanto a diversificação dos cenários reais de prática como o desenvolvimento do corpo docente. Este artigo aborda os desafios do ensino clínico nos cenários reais de prática e apresenta modelos e estratégias para professores e alunos qualificarem esta atividade dentro e fora do hospital.
\end{abstract}

Palavras-chave: Educação Médica. Hospitais de Ensino. Educação Médica Continuada. Preceptores. Ambulatório Hospitalar. Enfermaria.

\begin{abstract}
Clinical teaching in the real practice settings is an essential component of healthcare education. It ensures that students learn about professional practice, and stimulates effectively the development of clinical skills, particularly history and physical examination, communication, ethics, humanistic attitude and professionalism. Traditionally, wards and outpatient clinics, supervised by clinical staff (preceptors), are used for clinical teaching. However, despite the excellent training for professional practice, the majority of preceptors may not know sufficiently the principles of clinical teaching. Therefore, for an adequate clinical teaching, it is necessary to diversify and improve the real practice settings and faculty development. This article deals with the challenges of clinical teaching in the real practical settings and presents models and strategies for faculty and students qualify this activity inside and outside the hospital.
\end{abstract}

Keywords: Education, Medical. Hospitals, Teaching. Education, Medical, Continuing. Mentors. Outpatient Clinics, Hospital. Patients' Rooms.

1. Docente, Divisão de Emergências Clínicas, Departamento de Clínica Médica, Faculdade de Medicina de Ribeirão Preto - Universidade de São Paulo (FMRP-USP)

2. Médico Assistente do Hospital das Clínicas da FMRP-USP. Divisão de Nefrologia,

2. Docente, Divisão de Dermatologia, Departamento de Clínica Médica, FMRP-USP

4. Docente, Divisão de Infectologia, Departamento de Clínica Médica, FMRP-USP
Correspondência: Departamento de Clínica Médica, Faculdade de Medicina de Ribeirão Preto - Universidade de São Paulo

Artigo recebido em 25/11/2014 Aprovado para publicação em 18/12/2014 


\section{Principais pontos de interesse}

- O ensino clínico em cenários reais de prática é um componente essencial da educação na área da saúde. Ele propicia a aplicação do conhecimento teórico e é um dos mais efetivos meios para o desenvolvimento de habilidades clínicas, comunicação, ética, atitude humanística e profissionalismo.

- Tradicionalmente, as enfermarias e ambulatórios hospitalares são os locais mais utilizados para o ensino clínico, que é, na maioria das vezes, centrado em uma consulta real. Mais recentemente, há uma crescente tendência para a educação baseada na comunidade, onde os estudantes participam de práticas em unidades básicas e/ou de saúde da família. A todos estes locais denominaremos "cenários reais de prática", em contraste aos cenários de aprendizado simulado ou laboratórios de habilidades.

- Nos cenários reais de prática, o professor clínico (preceptor) desempenha um papel central de modelo para os alunos do ponto de vista técnico, humano e ético, o que dificilmente poderia ser aprendido e/ou ensinado apenas na sala de aula ou em ambientes de simulação.

- No ensino clínico, é crucial fornecer um feedback adequado.

- Dois modelos têm sido utilizados com sucesso no desenvolvimento e capacitação de professores clínicos, o Stanford Faculty Development model e o Microskills of teaching model (mais conhecido como o modelo do "one-minute preceptor"). Ambos podem ser utilizados nos mais diversos cenários de prática clínica e se baseiam em alterações e modulações comportamentais e valorização do feedback.

\section{Introdução}

Ensino clínico é definido como o ensino direcionado ao paciente e seus problemas e, geralmente, ocorre na presença deste, podendo acontecer em diversos cenários de ensino-aprendizado, tais como enfermarias, ambulatórios, unidades básicas de saúde, serviços de urgência e emergência, salas de reuniões, entre outras..$^{1,2,3}$

O ensino clínico consiste em componente essencial da educação profissional nos cursos da saúde, além de ser um dos mais efetivos meios para o desen- volvimento de habilidades clínicas. Particularmente na medicina, são nesses locais que os estudantes aprendem e praticam habilidades essenciais à prática profissional, tais como anamnese, exame físico, comunicação, ética, atitude humanística e profissionalismo, e também aplicam o conhecimento adquirido ao longo do curso. ${ }^{1-4}$ Em outras palavras, os estudantes aprendem sobre o exercício da profissão, o significado de ser médico.

Existem diversas evidências da importância do ensino clínico na formação de profissionais da saúde. O desenvolvimento das habilidades de comunicação e das relações interpessoais tem um impacto significativo no cuidado do paciente. ${ }^{5,6}$ Adicionalmente, a história clínica pode prover o diagnóstico em até $50 \%$ dos casos, e quando associada ao exame físico completo, o acerto diagnóstico pode atingir $70 \%$, ou até 90\% em certas circunstâncias. ${ }^{7}$

Tradicionalmente, as enfermarias e ambulatórios têm sido os locais mais utilizados para praticar o ensino clínico. Nesses cenários, o ensino é centrado em uma consulta real, onde os alunos acompanham uma consulta clínica, seguida por uma discussão que pode abordar, em diferente níveis, desde a anamnese às condições socioeconômicas do paciente. Porém, dependendo dos objetivos de aprendizagem e do nível de formação dos estudantes, sessões adicionais mais estruturadas podem ser necessárias para otimizar o aprendizado. ${ }^{3}$ Mais recentemente, observa-se uma crescente tendência de diversificação dos cenários de prática com a inclusão de atividades em unidades básicas de saúde ou em conjunto com profissionais das equipes de saúde da família. Esta tendência reflete uma recomendação da Organização Mundial da Saúde para a valorização da educação baseada na comunidade, ou seja, em ambientes mais próximos às comunidades e não apenas nos hospitais universitários.

No entanto, a despeito de sua relevância, numerosas barreiras têm sido identificadas na prática do ensino clínico. Na medicina, muitas vezes, os professores clínicos têm que lidar com a assistência ao doente e o ensino clínico, simultaneamente. ${ }^{8} \mathrm{Um}$ desafio adicional é o fato de que a maioria dos clínicos tende a estar melhor preparada para a prática médica, do que para o treinamento e supervisão de estudantes e residentes, especialmente no que diz respeito a métodos de ensino clínico. ${ }^{9}$ Adicionalmente, as oportunidades para o ensino clínico estão diminuindo frente aos modelos atuais de assistência à saúde, com períodos reduzidos de permanência dos pacientes nos 
serviços e excesso de confiança dos médicos nos recursos tecnológicos para o diagnóstico de seus pacientes. Essa situação tem propiciado uma geração de professores pouco familiarizados com alguns princípios básicos do ensino clínico, particularmente do ensino à beira do leito. ${ }^{1,4,10}$

Além disso, diversos desafios têm que ser considerados ao se programar o ensino clínico na enfermaria e/ou ambulatórios (Tabela 1), tais como: tempo que estas atividades demandam; professores clínicos geralmente têm outras atividades simultâneas (atividades clínicas, de pesquisa e administrativas); são atividades imprevisíveis, pouco reprodutíveis e difíceis de preparar; dificuldade para engajar alunos de diferentes níveis de formação e de diferentes profissões da saúde; pacientes com internações curtas ou que se recusam a participar; e ambiente físico nem sempre adequado. ${ }^{3}$

Nesse sentido, é fundamental minimizar as potenciais dificuldades e também estabelecer programas de capacitação docente. Algumas qualidades de um bom professor clínico encontram-se na Tabela 2. 3,11 Atualmente, dois modelos têm sido utilizados com sucesso na desenvolvimento de professores clínicos, o Stanford Faculty Development model ${ }^{12}$ e o Microskills of teaching model (the one-minute preceptor). ${ }^{13}$ Ambos se baseiam em alterações comportamentais e podem ser utilizados em diversos cenários clínicos. Suas principais características estão listadas na Tabela $3 .^{3}$ Para observar um exemplo do modelo one minute preceptor é possível acessar um vídeo no link a seguir: https://www.youtube.com/ watch? $\mathrm{v}=\mathrm{P} 0 \mathrm{XgABFzcgE}$.

Irby e colaboradores propõem uma estratégia rápida e efetiva, baseada em três passos, para melhorar o ensino clínico: planejamento; ensino; avaliação e reflexão. ${ }^{3,14}$ Por fim, o professor clínico também desempenha um papel de modelo para os alunos, tanto do ponto de vista técnico como humano e ético, o que dificilmente poderia ser aprendido e/ou ensinado na sala de aula ou em ambientes de simulação da prática. ${ }^{3}$

Tabela 1: Possíveis dificuldades do ensino clínico nos ambulatórios e enfermarias.

Dificuldades do ensino clínico nos ambulatórios

- Ambiente ocupado (falta de espaço físico)

- Pouco tempo para preparar a atividade e para o ensino

- Ausência de controle na distribuição e organização do tempo

- Diversos pacientes sendo atendidos ao mesmo tempo com vários alunos

- Interações curtas entre professor-aluno

- As demandas assistenciais geralmente têm prioridade

- Pacientes com vários problemas, dificultando focar o ensino em um problema

- Aprendizagem e assistência ocorrem simultaneamente

- Problemas orgânicos e psicossociais estão interligados

- Muitas vezes o tratamento é iniciado empiricamente (prova terapêutica)
Dificuldades do ensino clínico nas enfermarias

- Falta de disponibilidade de instalações físicas adequadas

- Dificuldade de definir os objetivos do ensino (imprevistos ocorrem com frequência)

- Equipe geralmente composta por alunos de diferentes níveis de formação

- Pacientes muito doentes ou que não querem participar

- Internações muito curtas

- Os professores, se "dominarem" o encontro, podem comprometer o relacionamento alunopaciente

- Insegurança em assumir erros na frente do paciente e/ou da equipe

- Dificuldade de envolver todos no aprendizado

- Alunos cansados, entediados e/ou sobrecarregados

\section{Professor deve ser um facilitador do aprendizado e não apenas transmitir informações.}

Adaptado de Ramani e colaboradores. ${ }^{3}$ 
Tabela 2: Características de um bom professor clínico

- Compartilhar sua paixão pelo ensino

- Ser claro, organizado, acessível, solidário e humano

- Ser capaz de estabelecer relações, fornecer orientação e feedback

- Ser íntegro e ter respeito pelos outros

- Demonstrar competência clínica

- Utilizar estratégias de planejamento e de orientação

- Utilizar diferentes métodos de ensino e roteiros

- Ensinar/facilitar o aprendizado dos princípios gerais relevantes para a prática profissional

- Adequar o ensino ao nível de conhecimento dos alunos

- Diversificar suas fontes de conhecimento

- Auxiliar os estudantes a produzirem síntese do seu aprendizado

- Regularmente fazer auto-avaliação e reflexão sobre sua prática como educador

Adaptado e ampliado, de Ramani e colaboradores. ${ }^{3}$

Tabela 3: Características de dois modelos para o desenvolvimento de professores clínicos

Stanford Faculty Development model

- Promover um clima positivo de aprendizagem

- Controlar a sessão

- Comunicar os objetivos

- Promover a compreensão e retenção

- Avaliação

- Feedback

- Promover a aprendizagem auto-dirigida

\section{Microskills of teaching model (one-minute preceptor)}

- Comprometimento com o caso

- Questionar as evidências concretas

- Ensinar as regras/ensinamentos gerais

- Reforçar o que está correto

- Corrigir o que está incorreto

Adaptado de Ramani e colaboradores. ${ }^{3}$

\section{Ensino clínico nas enfermarias}

A aplicação do conhecimento adquirido em aulas teóricas e as habilidades necessárias à prática clínica têm sido tradicionalmente alcançadas nas enfermarias ${ }^{15}$. No entanto, alguns desafios, listados na Tabela 1, precisam ser considerados na preparação dessas atividades. Além disso, nas instituições de saúde, as enfermarias nem sempre constituem locais ideais para o ensino, porém com algumas estratégias podem se tornar mais adequadas. ${ }^{15}$

A despeito dos obstáculos, o ensino clínico nas enfermarias provê valiosa oportunidade para demonstração e observação do exame físico, desenvolvimento de habilidades de comunicação e de um modelo de abordagem humanística do paciente. ${ }^{15}$ Ende sugere que, na preparação de um ensino efetivo nas enfermarias, os professores sempre façam as seguintes perguntas antes de cada encontro. ${ }^{3,16}$

1. O que você espera alcançar?

2. Qual é o seu ponto de vista/opinião?

3. Como os alunos serão envolvidos?

4. Como você alcançará os objetivos de aprendizagem de cada aluno?

5. Como as visitas serão organizadas?

6. Suas visitas são boas?

7. Como você organizará o tempo? 
O ensino na enfermaria deve respeitar a rotina dos cuidados ao paciente, procedimentos, refeições, higienização do leito e da enfermaria, e visitas. O uso de uma sala auxiliar de reuniões provê uma alternativa útil para a preparação prévia das atividades ou discussão posterior à visita de enfermaria. Esta situação favorece outro conceito atual e desejável no ensino e assistência nos cenários reais da prática, ou seja, a participação de outros profissionais e membros da equipe que poderão enriquecer a sessão atribuindo uma visão multiprofissional de assistência ao paciente. ${ }^{15}$

É importante ressaltar que o ensino clínico deve abordar os pacientes, e não exclusivamente as suas enfermidades. Esta atitude deve ser introduzida e processada no aprendizado do estudante, para que possa ser levada para a sua futura prática profissional. ${ }^{15}$

$\mathrm{O}$ ensino nas enfermarias pode ser analisado pela tríade paciente-estudante-tutor. ${ }^{15}$

\section{Pacientes}

Pacientes apropriados para o ensino clínico devem ser selecionados com cuidado e estar suficientemente bem para serem vistos pelos alunos. Por exemplo, para estudantes inexperientes, na medida do possível, o mais indicado é a seleção de pacientes comunicativos com histórias clínicas e achados físicos pouco complicados. Para este grupo de estudantes é valioso ter acesso também a pacientes com achados clínicos dentro do padrão da normalidade. Como a construção do conhecimento e da habilidade se faz através da prática, a exposição ao maior número de pacientes propicia ao estudante mais oportunidades de realizar a anamnese e o exame físico, e potencialmente maiores chances para o seu aprendizado e a sua capacidade de distinguir entre a normalidade e o que caracteriza a enfermidade. ${ }^{15}$

Os pacientes devem ser convidados para participar e também ter oportunidade de declinar. É ainda relevante a apresentação dos integrantes do grupo e da finalidade da respectiva atividade, para que os pacientes saibam o que será esperado deles, e que se sintam parte da discussão, com a possibilidade até de fornecerem um feedback aos estudantes. A maioria dos pacientes aprecia a experiência, sentindo e expressando satisfação por auxiliar os estudantes no aprendizado, ao mesmo tempo que têm seu problema avaliado em detalhes pelos "jovens doutores" e o professor. No ensino na enfermaria, em geral, são requeridos diversos pacientes, por períodos variáveis de tempo, sendo necessário estar atento às limitações e respeitar as necessidades de cada um deles. ${ }^{15}$

\section{Estudantes}

Em geral, o número ideal para o ensino clínico é de dois a cinco estudantes. Eles devem estar apropriadamente vestidos com aventais ou roupas brancas e identificados com crachás. ${ }^{17}$ É esperado que se apresentem aos profissionais da saúde e aos pacientes, explicitem a proposta de sua visita e que se comportem apropriadamente. Uma prévia comunicação ao início da atividade deve explicitar aos estudantes a proposta da sessão e os objetivos a serem atingidos. ${ }^{15}$

Qualquer informação sobre as condições do paciente deve ser adequada ao nível do estudante. Ao refletir sobre suas experiências, os estudantes têm a compreensão da importância do ensino na enfermaria no desenvolvimento de habilidades clínicas. Porém, inicialmente, alguns estudantes possam se sentir intimidados por um ambiente não familiar, pela proximidade de outros profissionais da saúde ou por ter que realizar perguntas pessoais a um estranho. Assim, eles podem se sentir ansiosos, inseguros do seu conhecimento ou habilidades clínicas e receosos da crítica do tutor sobre as suas inadequações. Com isso, alguns estudantes adotam posições de retaguarda no grupo para evitar participação, em contraste, estudantes seguros podem monopolizar a conversa com o paciente. Os tutores devem estar atentos para compensar este desequilíbrio, assegurando a participação de todos os estudantes e aliviando a ansiedade. ${ }^{15}$

\section{Tutores (preceptores)}

É fundamental que os tutores (preceptores) tenham ciência de que eles são um modelo para os estudantes. Assim, mais do que nunca estes preceptores devem demonstrar conhecimento, habilidades e atitudes compatíveis com os mais altos valores da sua profissão. Deste modo, mais que proficiência clínica o preceptor deve ser a referência na enfermaria nos quesitos pontualidade, entusiasmo, profissionalismo, cordialidade e empatia com os pacientes, estudantes e equipe de saúde. ${ }^{15}$

Por se tratar de um cenário com relativa estabilidade, as práticas clínicas na enfermaria devem ser cuidadosamente planejadas pelos preceptores, de modo que os objetivos de aprendizagem possam ser 
alcançados plenamente. Outro recurso que pode ser utilizado, especialmente para o ensino de alunos iniciantes, é o de visitas no período da tarde, quando a rotina da enfermaria é mais tranquila, em contraste com as atividades do período da manhã que devem ser direcionadas para estudantes do internato e residentes que assumem o cuidado do paciente com maior grau de autonomia, porém sempre supervisionados por preceptores. ${ }^{15}$

\section{Ensino clínico em ambulatórios}

Nos últimos anos, o desenvolvimento da medicina, fatores econômicos e sociais levaram pacientes de alta complexidade, previamente tratados em regime hospitalar, para um regime ambulatorial. ${ }^{18}$ Essa tendência mundial também ocorreu no Brasil e de forma intensificada pela redução do número de leitos hospitalares frente a uma população em crescimento e com o aumento da expectativa de vida. Esta mudança transformou o cenário ambulatorial em um ambiente mais diversificado, em contraposição ao hospitalar, que hoje está mais restrito a pacientes de maior gravidade e complexidade. Apesar de mais diversificados, os pacientes atendidos nos ambulatórios encontram-se estáveis e, na sua maioria, dispostos a serem atendidos por alunos.

$\mathrm{O}$ ambulatório permite ainda o seguimento de pacientes com doenças crônicas cujo controle, na maioria das vezes, não necessita de internação, tornando esse ambiente único para o contato com algumas condições clínicas que não são vistas no regime hospitalar. Além disso, permite uma atividade mais próxima a que será realizada por muitos alunos após o final da graduação.

Ambulatórios de hospitais universitários contam com um amplo número de pacientes com a mesma condição clínica. ${ }^{19}$ Isso possibilita que o tutor escolha pacientes com grau de complexidade apropriado para o nível de aprendizado do aluno, permitindo o contato com vários casos de uma mesma doença e suas diversas apresentações clínicas.

No ensino clínico nas enfermarias, o tutor reserva um horário em sua agenda para realizar a atividade com o aluno e, muitas vezes, essa atividade não influencia na condução do caso. Livre da necessidade de tomada de decisão clínica, a atenção e o tempo do tutor ficam direcionados ao aluno e ao ensino. Por outro lado, quando a atividade de ensino é deslocada para fora das enfermarias, o aluno é escalado para um ambulatório em que o tutor, geralmente, já exerce suas atividades de assistência. Neste caso, a interação entre tutor e aluno tem duas funções simultâneas: definição de conduta e ensino clínico. ${ }^{20}$

Os ambulatórios dos hospitais universitários apresentam número variado de pacientes e, muitas vezes, uma equipe em diferentes fases de aprendizado, de alunos de graduação a residentes de especialidades. O número de pacientes a serem atendidos frequentemente obriga a redução do tempo dedicado à discussão com alunos para poucos minutos por paciente atendido. Com isso, o fator tempo pode tornar a discussão mais objetiva e o aprendizado mais passivo. Essa postura passiva foi descrita inicialmente por Foley em 1979 e, décadas depois, por Irby em 1995. ${ }^{21,22}$

Assim, os alunos tendem apenas a apresentar o caso e se tornam descritores, raramente levantando questionamentos ou propondo um plano de ação para a situação em questão. Essa postura parece ser mantida mesmo quando o tutor discute o caso com um residente de especialidade. Wolpaw e colaboradores evidenciaram que residentes mais experientes são mais efetivos para apresentar um caso em ambulatório, sendo capaz de transmitir a mesma informação em menos tempo. No entanto, mantêm a mesma postura passiva agindo mais como descritores de casos do que residentes ativos e questionadores. ${ }^{23}$ Essa postura sugere que o comportamento de descritor pode ter sido estimulado pelos preceptores durante a residência médica.

É fundamental, portanto, que o preceptor estimule o aluno ao questionamento. Assim, ele deve solicitar que o aluno elabore uma hipótese diagnóstica, sugira diagnósticos diferenciais e proponha um plano de ação. Além disso, o aluno precisa ser estimulado a estudar o assunto visto no ambulatório o mais precoce possível. O ideal é que ele levante uma questão relevante sobre o caso, com o auxílio do preceptor, e tente fazer um estudo dirigido sobre este tópico. ${ }^{20}$

Esse modelo de abordagem em seis passos, descrita por Wolpaw e colaboradores, é conhecida pelo mnemônico, em inglês, SNAPPS: 1. Summarize briefly history and exam findings $=$ resuma a história e os achados do exame físico; 2. Narrow the differential diagnosis to two or three relevant possibilities $=$ resuma o diagnóstico diferencial em duas ou três possibilidades relevantes; 3 . Analyse the differential by comparing and contrasting the possibilities $=$ analise o diagnóstico diferencial, comparando e contrastando as possibilidades; 4. Probe 
the preceptor by asking questions about uncertainties, difficulties, or alternative approaches $=$ questione $\mathrm{o}$ preceptor sobre suas dúvidas, incertezas e abordagens alternativas; 5. Plan management for the patient's medical issues = planeje o cuidado do paciente; 6 . Select a case-related issue for self-directed learning = selecione um aspecto do caso para servir de guia para seu auto-estudo. ${ }^{20}$ Desta maneira, o aluno será estimulado a estudar todos os dias de forma sistemática fazendo conexão entre teoria e prática. Para observar um exemplo da utilização desse modelo (SNAPPS) acesse o link: https://www.youtube.com/ watch? $\mathrm{v}=\mathrm{BPNOdPKUFDE}$

\section{Ensino clínico nas unidades de saúde da atenção básica ou de saúde da família}

Este é um cenário mais recente na formação dos profissionais da saúde, em especial do médico. Existe uma recomendação formal para gestores acadêmicos, que são responsáveis pelo desenho do currículo dos cursos de medicina, no sentido de diversificar cenários de práticas e privilegiar espaços fora do hospital. O termo genérico para esta iniciativa é educação baseada na comunidade, e recomenda-se que este tipo de atividade seja implementada desde os primeiros anos do curso de medicina, proporcionando ao estudante o conhecimento da comunidade em que vive o paciente, bem como as unidades básicas de saúde, responsáveis pelo primeiro atendimento e também pelo maior volume de consultas médicas no sistema de saúde. Os estudantes costumam valorizar muito atividades com essas características. $\mathrm{O}$ maior desafio neste caso é a garantia de preceptores, já que os professores clínicos estão acostumados a trabalhar em hospitais e ambulatórios especializados. Como cenário de aprendizado, a atenção básica guarda uma série de semelhanças e desafios com o ensino em

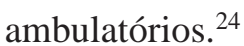

\section{Ensino clínico em serviços de urgência e emergência}

A medicina de emergência é uma área que objetiva o atendimento ágil, eficaz e tempo-dependente em situações de agravo agudo à saúde das pessoas, pressupondo hierarquização do sistema de saúde. A inserção da emergência como disciplina curricular, embora ainda incipiente no Brasil, vem ganhando espaço especialmente após a publicação das diretrizes curriculares dos cursos de graduação em medicina que reforça a necessidade do ensino-aprendizagem sobre urgências e emergências durante a graduação. Esta é uma área nova que deverá contar com cenários reais de prática, mas que, por ter situações com risco eminente de morte, todas práticas devem ser supervisionadas de perto e muitas vezes precedidas por treinamento em simuladores (vide capítulos de práticas simuladas). ${ }^{25}$

\section{Conclusão}

O ensino em cenários reais de prática é uma atividade essencial para a formação da identidade e capacitação profissional e requer um planejamento cuidadoso levando-se em consideração os recursos disponíveis e os objetivos de aprendizagem que se espera alcançar com os estudantes. Muitos profissionais da saúde experientes não estão preparados para atuarem como professores clínicos (preceptores) de estudantes e ou residentes, o que pode ser contornado se existirem condições para treinamento e capacitação. Finalmente, é importante lembrar que não será possível oferecer um bom aprendizado clínico se não houver uma prática de boa qualidade em todos níveis de atendimento, diversificação de cenários, organização do sistema de saúde, profissionais capacitados e adequada infraestrutura. Esta é a base sobre a qual é possível treinar bons profissionais na área da saúde tanto para prática profissional como para atuarem como preceptores na formação da futura geração.

\section{Referências}

1. Janicik RW, Fletcher KE. Teaching at the bedside: a new model. Med Teach. 2003;25:127-30.

2. Spencer J. Learning and teaching in the clinical environment. BMJ. 2003;326:591-4.

3. Ramani S, Leinster S. AMEE Guide no. 34: Teaching in the clinical environment. Med Teach. 2008;30:347-64.

4. Ramani S. Twelve tips to improve bedside teaching. Med Teach. 2003;25:112-5.

5. Rider EA, Keefer $\mathrm{CH}$. Communication skills competencies: definitions and a teaching toolbox. Med Educ. 2006;40:624-9.

6. Langlois JP, Thach S. Teaching at the bedside. Fam Med. 2000;32:528-30.

7. Peters $\mathrm{M}$, Ten Cate $\mathrm{O}$. Bedside teaching in medical education: a literature review. Perspect Med Educ. 2014;3:76-88. 
8. Prideaux D, Alexander H, Bower A, Dacre J, Haist S, Jolly B et al. Clinical teaching: maintaining an educational role for doctors in the new health care environment. Med Educ. 2000;34:820-6.

9. Steinert Y. Staff development for clinical teachers. The Clinical Teacher. 2005;2:104-10.

10. Salam A, Siraj HH, Mohamad N, Das S, Rabeya Y. Bedside teaching in undergraduate medical education: issues, strategies, and new models for better preparation of new generation doctors. Iran J Med Sci. 2011;36:1-6.

11. Irby DM, Papadakis M. Does good clinical teaching really make a difference? Am J Med. 2001;110:231-2.

12. Skeff KM. Enhancing teaching effectiveness and vitality in the ambulatory setting. J Gen Intern Med. 1988;3(2 Suppl):S2633.

13. Neher JO, Gordon KC, Meyer B, Stevens N. A five-step "microskills" model of clinical teaching. J Am Board Fam Pract. 1992:5:419-24.

14. Irby DM, Bowen JL. Time efficient strategies for learning and performance. The Clinical Teacher. 2004;1:23-8.

15. Dent J, Harden RM. A practical guide for medical teachers: Elsevier Health Sciences; 2013.

16. Ende J. What if Osler were one of us? Inpatient teaching today. J Gen Intern Med. 1997;12 Suppl 2:S41-8.

17. Yonekura CL, Certain L, Karen SKK, Alcântara GAS, Ribeiro LG, Rodrigues-Júnior AL, et al. Impressões de pacientes, médicos e estudantes de Medicina quanto a aparência dos médicos. Rev Assoc Med Bras. 2013;59:452-9.
18. Brook RH, Kamberg CJ, McGlynn EA. Health system reform and quality. JAMA. 1996;276:476-80.

19. O’Malley PG, Kroenke K, Ritter J, Dy N, Pangaro L. What learners and teachers value most in ambulatory educational encounters: a prospective, qualitative study. Acad Med. 1999;74:186-91.

20. Wolpaw TM, Wolpaw DR, Papp KK. SNAPPS: a learnercentered model for outpatient education. Acad Med. 2003;78:893-8.

21. Foley R, Smilansky J, Yonke A. Teacher-student interaction in a medical clerkship. J Med Educ. 1979;54:622-6.

22. Irby DM. Teaching and learning in ambulatory care settings: a thematic review of the literature. Acad Med. 1995;70:898-931.

23. Wolpaw TM, Glover PB, Papp KK. An exemplary model of learning in the rheumatology outpatient setting. Arthritis Rheum 2001; 44: S208

24. Bollela VR, Passos ADC, Forster AC, Dal Fabbro AL, F. B-R, C. RFJ, et al. Community-Based Education experience at the Faculty of Medicine, Ribeirão Preto, University of São Paulo (FMRP-USP) - Brazil In: Talaat W, Ladhani Z, editors. Community Based Education in Health Professions: Global Perspectives World Health Organization (WHO); 2014. p. 62-88.

25. Pazin-Filho A, Scarpelini S. Medicina de Emergência na FMRP-USP-Você pode fugir, mas não há como se esconder! Medicina (Ribeirao Preto Online). 2010;43:432- 43. 